doi:10.13108/2015-7-2-106

\title{
SPACE MOTIONS WITH THE LINEAR FIELD OF VELOCITY WITHOUT DIVERGENCE
}

\section{S.V. KHABIROV}

\begin{abstract}
We obtain the formulae defining all possible motions of continuous medium without divergence and with the linear field of the velocity. The field is either linear w.r.t. the time or the matrix has constant singular numbers.
\end{abstract}

Keywords: dynamics without divergence, linear field of velocity, singular numbers of the matrix.

Mathematics Subject Classification: 35B06, 35Q35

\section{INTRODUCTION}

In classification of solid state motions with a linear field of velocity [1, 2], there appear a special case of motion without divergence when the divergence of the velocity vanishes. One deals with an overdetermined system of ordinary differential equations and its compatibility is to be studied. For the general motion, this situation corresponds, for instance, to the solutions of compressible liquid equations with zero divergence [3]

$$
\begin{gathered}
\vec{u}_{t}+(\vec{u} \cdot \nabla) \vec{u}+\rho^{-1} \nabla p=0, \quad \nabla \cdot \vec{u}=0, \\
\rho_{t}+\vec{u} \cdot \nabla \rho=0, \quad p_{t}+\vec{u} \cdot \nabla p=0,
\end{gathered}
$$

where $\vec{u}, p$ and $\rho$ are the velocity, pressure and density. System (0.1) is valid for each state equation determining the entropy. In the spatial case, overdetermined system (0.1) is not reduced to involution and the arbitrariness in solutions is not known (how to impose initial data).

Physical meaning of solutions with zero divergence is as follows: given a bounded volume, the inflow of the liquid equals to the outflow.

Plane motions without divergence in a linear field of velocity are listed in [4]. The aim of this work is to find all solutions to equations (0.1) with linear field of velocity in the spatial case. As in the planar case, we obtain solutions with linear in time matrix or the matrix has constant singular numbers.

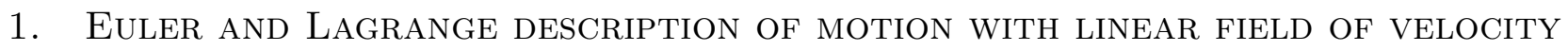

Solutions to system (0.1) with linear field of velocity

$$
\vec{u}=A(t) \vec{x}+\vec{u}_{0}(t)
$$

define an Euler submodel of the overdetermined system of ordinary differential equations

$$
\operatorname{tr} A=0, \quad A^{\prime}+A^{2}=B, \quad B^{\prime}+A^{T} B+B A=0,
$$

S.V. Khabirov, Space motions with linear feild of Velocity Without divergence.

(C).V. KhaBirov, 2015.

The work is supported by RFBR (12-01-00648, 14-01-97027), by the grant of the President of Russia for Leading Scientific Schools (NSh-6706.2012.1), by grant no. 11.G34.31.0042 of the Goverment of Russia under the law no. 220 .

Submitted May 16, 2014. 


$$
\vec{u}_{0}^{\prime}+A \vec{u}_{0}=\vec{a}, \quad \vec{a}^{\prime}+A^{T} \vec{a}+B \vec{u}_{0}=0 .
$$

Lagrange variables $t, \vec{\xi}$ are introduced as solution to the problem

$$
\vec{x}^{\prime}=A(t) \vec{x}+\overrightarrow{u_{0}}, \quad \vec{x}\left(t_{0}\right)=\vec{\xi},
$$

and are defined by the formulae

$$
\vec{x}=\overrightarrow{x_{0}}(t)+M(t) \vec{\xi}, \quad M\left(t_{0}\right)=I, \quad \overrightarrow{x_{0}}\left(t_{0}\right)=0 .
$$

Here $I$ is the unit matrix. Identities (1.3), (1.4) imply the formulae for matrices $A, B$ and vectors $\overrightarrow{u_{0}}, \vec{a}$ in terms of matrix $M$ and vector $\overrightarrow{x_{0}}$ :

$$
\begin{gathered}
A=M^{\prime} M^{-1}, \quad B=M^{\prime \prime} M^{-1}, \\
\vec{u}_{0}=\vec{x}_{0}^{\prime}-M^{\prime} M^{-1} \vec{x}_{0}, \quad \vec{a}=\vec{x}_{0}^{\prime \prime}-M^{\prime \prime} M^{-1} \vec{x}_{0} .
\end{gathered}
$$

Equations (1.2) yield the equations of Lagrange submodel

$$
M^{T} M^{\prime \prime}=C=S_{0}+E<\vec{\omega}_{0}>, \quad M^{T} \vec{x}_{0}^{\prime \prime}=\vec{c}, \quad|M|=1,
$$

where $C$ and $\vec{c}$ are constant matrix and vector as a result of integration, $S_{0}=\left\|s_{i j}^{0}\right\|$ and $E<\vec{\omega}_{0}>$ are symmetric and anti-symmetric part of matrix $C$. Here we have employed the identity

$$
|M|^{\prime}=|M| \operatorname{tr}\left(M^{\prime} M^{-1}\right) .
$$

Initial conditions to system (1.5) read as

$$
M\left(t_{0}\right)=I, \quad M^{\prime}\left(t_{0}\right)=M_{1}=S_{1}+E<\vec{\omega}_{1}>, \quad \operatorname{tr} S_{1}=0,
$$

where $S_{1}$ and $E<\vec{\omega}_{1}>$ are symmetric and anti-symmetric parts of matrix $M_{1}$. Anti-symmetric matrices are defined via vectors by the formula

$$
E<\vec{\omega}_{i}>=\left\|\begin{array}{ccc}
0 & -\omega_{i}^{3} & \omega_{i}^{2} \\
\omega_{i}^{3} & 0 & -\omega_{i}^{1} \\
-\omega_{i}^{2} & \omega_{i}^{1} & 0
\end{array}\right\| .
$$

In Lagrange variables, equations (0.1) become

$$
p_{t}=0, \quad \rho_{t}=0, \quad \nabla_{\xi} p=-\rho(\vec{\xi})(C \vec{\xi}+\vec{c}) .
$$

The compatibility conditions of system (1.7) are as follows:

$$
\nabla_{\xi} \rho \times\left(S_{0} \vec{\xi}+\vec{c}+\vec{\omega}_{0} \times \vec{\xi}\right)=2 \rho \vec{\omega}_{0} .
$$

It yields the equations for a motion with a variable density

$$
\begin{gathered}
\vec{\omega}_{0} \cdot \nabla_{\xi} \rho=0, \quad \nabla_{\xi} \rho \times\left(S_{0} \vec{\xi}+\vec{c}\right)+\vec{\omega}_{0}\left(\vec{\xi} \cdot \nabla_{\xi} \rho-2 \rho\right)=0, \\
\nabla_{\xi} \rho\left(\vec{\omega}_{0} \cdot\left(S_{0} \vec{\xi}+\vec{c}\right)\right)=0 \quad \Rightarrow \quad S_{0} \vec{\omega}_{0}=0, \quad \vec{\omega}_{0} \cdot \vec{c}=0 .
\end{gathered}
$$

If $\vec{\omega}_{0}=0$, by equations (1.8) and (1.7) we get the expressions for the density and pressure

$$
\rho=P^{\prime}(J), \quad p=P_{0}-P(J), \quad J=2^{-1} \vec{\xi} \cdot S_{0} \vec{\xi}+\vec{c} \cdot \vec{\xi}
$$

Hereinafter $P_{0}$ is a constant, $P(J)$ is an arbitrary function.

If $\vec{\omega}_{0} \neq 0$, for instance, $\omega_{0}^{1} \neq 0$, the scalar equation in (1.8) has the general solution

$$
\rho=\rho(\alpha, \beta), \quad \alpha=\xi^{2}-\omega_{0}^{2}\left(\omega_{0}^{1}\right)^{-1} \xi^{1}, \quad \beta=\xi^{3}-\omega_{0}^{3}\left(\omega_{0}^{1}\right)^{-1} \xi^{1},
$$

and vector equation (1.8) is reduced to

$$
\rho_{\alpha}\left(c^{3}+\left(s_{23}^{0}+\omega_{0}^{2}\right) \alpha+s_{33}^{0} \beta\right)+\rho_{\beta}\left(-c^{2}-s_{22}^{0} \alpha-\left(s_{23}^{0}-\omega_{0}^{1}\right) \beta\right)=-2 \omega_{0}^{1} \rho .
$$


If $\triangle=s_{22}^{0} s_{33}^{0}-\left(s_{23}^{0}\right)^{2}+\left(\omega_{0}^{1}\right)^{2} \neq 0$, it is possible to shift independent variables $\alpha, \beta$ by constants $\alpha_{0}, \beta_{0}$

$$
\begin{gathered}
\alpha=\alpha_{1}+\alpha_{0}, \quad \beta=\beta_{1}+\beta_{0}, \\
\alpha_{0}=\triangle^{-1}\left(c^{3}\left(s_{23}^{0}-\omega_{0}^{1}\right)-c^{2} s_{33}^{0}\right), \quad \beta_{0}=\triangle^{-1}\left(c^{2}\left(s_{23}^{0}+\omega_{0}^{1}\right)-c^{3} s_{22}^{0}\right)
\end{gathered}
$$

so that equation (1.10) admits a uniform dilation w.r.t. variables $\alpha_{1}$ and $\beta_{1}$. Introducing then an invariant $K=\beta_{1} \alpha_{1}^{-1}$ as a new independent variable together with $\alpha_{1}$, we obtain an integrable equation. Its solution and the solution to equation (1.7) are represented as

$$
\begin{gathered}
\rho=R^{\prime \prime}(J) \exp \left(2 \omega_{0}^{1} \int P^{-1}(K) d K\right), \quad J=\alpha_{1}^{2} P(K) \exp \left(2 \omega_{0}^{1} \int P^{-1}(K) d K\right), \\
P(K)=s_{33}^{0} K^{2}+2 s_{23}^{0} K+s_{22}^{0}, \quad p=P_{0}+R(J)-J R^{\prime}(J), \quad R^{\prime \prime}>0,
\end{gathered}
$$

where $R(J)$ is an arbitrary function.

If $\left(s_{22}^{0}\right)^{-1}\left(s_{23}^{0}-\omega_{0}^{1}\right)=s_{33}^{0}\left(s_{23}^{0}+\omega_{0}^{1}\right)^{-1}=\lambda \neq 0$, the change $K=\alpha+\beta \lambda$ leads us to integrable equation (1.10). Its solution and the solution to equation (1.7) read as

$$
\begin{gathered}
\rho=\frac{J P^{\prime}(J)}{2 \omega_{0}^{1} K+c^{3}-\lambda c^{2}}, \quad J=\left|2 \omega_{0}^{1} K+c^{3}-\lambda c^{2}\right|^{n} \exp \left(\beta+2^{-1}\left(\omega_{0}^{1}\right)^{-1} s_{22}^{0} K\right), \\
p=P_{0}-P(J), \quad n=4^{-1}\left(\omega_{0}^{1}\right)^{-2}\left(\left(s_{23}^{0}+\omega_{0}^{1}\right) c^{2}-s_{22}^{0} c^{3}\right) .
\end{gathered}
$$

If $\lambda=0, s_{33}^{0}=0, s_{23}^{0}=\omega_{0}^{1}$, formulae (1.12) remain true in this case as well.

For a constant density, it follows from (1.7) that

$$
\vec{\omega}_{0}=0, \quad p=P_{0}-\rho_{0}\left(\vec{c} \cdot \vec{\xi}+\vec{\xi} \cdot S_{0} \vec{\xi}\right)
$$

Thus, formulae for the density and the pressure are obtained in terms of Lagrange variables.

\section{SOLUTIONS WITH LINEAR MATRIX OF LINEAR FIELD OF VELOCITY}

If $C=0$, then matrix $M$ is linear in time $t\left(t_{0}=0\right)$

$$
M=I+t M_{1} \text {. }
$$

Equation $|M|=1$ implies the identities $\left|M_{1}\right|=0, \operatorname{tr} M_{1}=0, \operatorname{tr} M_{1}^{2}=0$, i.e., matrix $M_{1}$ is nilpotent $M_{1}^{3}=0$,

$$
M^{-1}=I-M_{1} t+M_{1}^{2} t^{2}, \quad A=M^{\prime} M^{-1}=M_{1}-M_{1}^{2} t .
$$

By choosing the coordinate system, we reduce matrix $M_{1}$ to its Jordan form. All the eigenvalues of matrix $M_{1}$ are zero. Two types of Jordan matrices are possible:

$$
\begin{aligned}
\text { 1) } M_{1}= & \left\|\begin{array}{lll}
0 & 0 & 0 \\
0 & 0 & 1 \\
0 & 0 & 0
\end{array}\right\|=A, \quad \vec{u}_{0}=\| \begin{array}{c}
C_{1} t+C_{2} \\
C_{6}-\left(\begin{array}{c}
\left.C_{3}+6 C_{5}\right) t-C_{4} t^{2}-C_{5} t^{3} \\
C_{3}+2 C_{4} t+3 C_{5} t^{2}
\end{array} \| ;\right.
\end{array} \text { 2) } M_{1}=\left\|\begin{array}{lll}
0 & 1 & 0 \\
0 & 0 & 1 \\
0 & 0 & 0
\end{array}\right\|, \quad A=\left\|\begin{array}{ccc}
0 & -t \\
0 & 0 & 1 \\
0 & 0 & 0
\end{array}\right\|, \\
\vec{u}_{0}= & \left\|\begin{array}{c}
C_{6}+\left(12 C_{1}-C_{5}\right) t+\left(C_{4}-3 C_{2}\right) t^{2}+\left(2 C_{1}+C_{3}\right) t^{3}+C_{2} t^{4}-C_{1} t^{5} \\
C_{5}+\left(6 C_{2}-C_{4}\right) t-\left(6 C_{1}+C_{3}\right) t^{2}-C_{2} t^{3}+C_{1} t^{4} \\
C_{4}+2 C_{3} t+3 C_{2} t^{2}-4 C_{1} t^{3}
\end{array}\right\| .
\end{aligned}
$$

Here $C_{i}$ are constants.

In what follows, matrix $C$ is non-zero. 


\section{INTEGRALS FOR LAGRAGNE MODEL AND ITS MODIFICATION}

The symmetric part of matrix equation of model (1.5) reads as

$$
M^{T} M^{\prime \prime}+M^{\prime \prime T} M=2 S_{0} .
$$

Anti-symmetric part of matrix equation (1.5) is integrable

$$
M^{T} M^{\prime}-M^{\prime T} M=2 E<\vec{\omega}>, \quad \vec{\omega}=t \vec{\omega}_{0}+\vec{\omega}_{1} .
$$

Here initial conditions (1.6) are taken at $t_{0}=0$.

Equations (1.5) admit a translation along $t$. We shift initial conditions at $\vec{\omega}_{0} \neq 0$ into $t_{0}=-\left(\vec{\omega}_{0} \cdot \vec{\omega}_{1}\right)\left|\vec{\omega}_{0}\right|^{-2}$, so that the transformed vector $\vec{\omega}_{1}^{\prime}=t_{0} \vec{\omega}_{0}+\vec{\omega}_{1}$ is orthogonal to vector $\vec{\omega}_{0}$. It is equivalent to the identity

$$
\vec{\omega}_{0} \cdot \vec{\omega}_{1}=0
$$

at $t_{0}=0$.

Matrix $M$ can be represented as the product of an orthogonal and symmetric matrices

$$
\begin{gathered}
M=Q \Lambda, \quad Q Q^{T}=I, \quad \Lambda^{T}=\Lambda, \quad|\Lambda|=1, \quad|Q|=1, \\
\Lambda(0)=I, \quad Q(0)=I, \quad \Lambda^{\prime}(0)=S_{1} .
\end{gathered}
$$

Matrix $E<\vec{q}>=Q^{T} Q^{\prime}$ is anti-symmetric, $\vec{q}(0)=\vec{\omega}_{1}$.

We write integral (3.2) as

$$
\Lambda \Lambda^{\prime}-\Lambda^{\prime} \Lambda+2 E<\Lambda^{-1} \vec{q}>=2 E<\vec{\omega}>.
$$

It determines matrix

$$
E<\vec{q}>=E<\Lambda \vec{\omega}>+2^{-1}\left(\Lambda^{-1} \Lambda^{\prime}-\Lambda^{\prime} \Lambda^{-1}\right) .
$$

By (3.4), equation (3.1) is represented as

$$
\left(\Lambda^{2}\right)^{\prime \prime}=\left(\left(\Lambda^{2}\right)^{\prime}-2 E<\vec{\omega}>\right) \Lambda^{-2}\left(\left(\Lambda^{2}\right)^{\prime}+2 E<\vec{\omega}>\right)+4 S_{0} .
$$

Initial conditions (1.6) are written as

$$
\Lambda^{2}(0)=I, \quad\left(\Lambda^{2}\right)^{\prime}(0)=2 S_{1}, \quad \operatorname{tr} S_{1}=0, \quad\left(\Lambda^{2}\right)^{\prime \prime}(0)=2\left(M_{1}^{T} M_{1}+S_{0}\right) .
$$

It is convenient to represent symmetric matrix $\Lambda^{2}$ as

$$
\Lambda^{2}=O D O^{T}, \quad D=\operatorname{diag}\left(\lambda_{1}, \lambda_{2}, \lambda_{3}\right), \quad O O^{T}=I, \quad|O|=1 .
$$

By choosing the coordinate system, the initial conditions can be represented as

$$
O(0)=I, \quad D(0)=I, \quad D^{\prime}(0)=2 S_{1} .
$$

The rotation matrix $O$ can be defined by the vector of angular velocity $\vec{\sigma}$

$$
O^{\prime}=O E<\vec{\sigma}>\text {. }
$$

Scalar equation (1.5) reduces to a simple form

$$
|D|=\lambda_{1} \lambda_{2} \lambda_{3}=1 \text {. }
$$




\section{Simplest SOLUTiON}

System (3.5) is compatible. Indeed, as $\Lambda^{2}=I$, identities (3.5), (3.6) imply compatibility conditions for the parameters

$$
S_{0}=E<\vec{\omega}>^{2}, \quad S_{1}=0 \Rightarrow \vec{\omega}_{0}=0, \quad S_{0}=E<\vec{\omega}_{1}>^{2} \neq 0 .
$$

For these values of parameters, identity (3.4) yield the relations

$$
Q^{\prime}=Q E<\vec{\omega}_{1}>, \quad M=Q, \quad O(0)=I .
$$

Matrix equation is integrable $Q=\exp \left(t E<\vec{\omega}_{1}>\right)$ and it follows that matrix $Q$ and $E<\vec{\omega}_{1}>$ commute.

By means of formula

$$
E<\vec{\omega}_{1}>^{2 k}=(-1)^{k}\left|\vec{\omega}_{1}\right|^{2 k}\left(I-\frac{\vec{\omega}_{1}}{\left|\vec{\omega}_{1}\right|} \otimes \frac{\vec{\omega}_{1}}{\left|\vec{\omega}_{1}\right|}\right)
$$

we calculate the matrix exponent

$$
\begin{aligned}
M=\exp \left(t E<\vec{\omega}_{1}>\right)= & I \cos \left(t\left|\vec{\omega}_{1}\right|\right)+\sin \left(t\left|\vec{\omega}_{1}\right|\right) E<\frac{\vec{\omega}_{1}}{\left|\vec{\omega}_{1}\right|}> \\
& +\left(1-\cos \left(t\left|\vec{\omega}_{1}\right|\right)\right) \frac{\vec{\omega}_{1}}{\left|\vec{\omega}_{1}\right|} \otimes \frac{\vec{\omega}_{1}}{\left|\vec{\omega}_{1}\right|} .
\end{aligned}
$$

Vector equation (1.5) becomes

$$
\vec{x}_{0}^{\prime \prime}=\vec{c} \cos \left(t\left|\vec{\omega}_{1}\right|\right)+\left(1-\cos \left(t\left|\vec{\omega}_{1}\right|\right)\right) \frac{\vec{\omega}_{1}}{\left|\vec{\omega}_{1}\right|^{2}}\left(\vec{\omega}_{1} \cdot \vec{c}\right)+\sin \left(t\left|\vec{\omega}_{1}\right|\right) \frac{\vec{\omega}_{1}}{\left|\vec{\omega}_{1}\right|} \times \vec{c} .
$$

Integrating, we get

$$
\begin{aligned}
\vec{x}_{0}= & -\frac{\vec{c}}{\left|\vec{\omega}_{1}\right|^{2}} \cos \left(t\left|\vec{\omega}_{1}\right|\right)+\left(2^{-1} t^{2}+\frac{\cos \left(t\left|\vec{\omega}_{1}\right|\right)}{\left|\vec{\omega}_{1}\right|^{2}}\right) \frac{\vec{\omega}_{1}}{\left|\vec{\omega}_{1}\right|^{2}} \vec{\omega}_{1} \cdot \vec{c} \\
& -\sin \left(t\left|\vec{\omega}_{1}\right|\right) \frac{\vec{\omega}_{1}}{\left|\vec{\omega}_{1}\right|^{3}} \times \vec{c}+t\left(\vec{c}_{1}+\frac{\vec{\omega}_{1} \times \vec{c}}{\left|\vec{\omega}_{1}\right|^{2}}\right)+\frac{\vec{c}}{\left|\vec{\omega}_{1}\right|^{2}}-\frac{\vec{\omega}_{1}}{\left|\vec{\omega}_{1}\right|^{4}} \vec{\omega}_{1} \cdot \vec{c},
\end{aligned}
$$

where $\vec{c}$ and $\vec{c}_{1}$ are constant vectors.

Vector $\vec{u}_{0}$ is determined via expression (1.1) by the formula

$$
\vec{u}_{0}=\vec{x}_{0}^{\prime}-\vec{\omega}_{1} \times \vec{x}_{0} .
$$

If $\Lambda^{2}=I$, the motion of the media goes as the solid state motion [6, Sect. 1].

The statement is valid: condition $S_{0}=E<\vec{\omega}_{1}>^{2}$ is necessary and sufficient for the existence of the simplest solution. It can be proven straightforwardly, but it is also implied by the following statement on absence of other solutions.

\section{ON ABSENCE OF OTHER SOUTIONS}

Apart from solutions with linear in time matrix $M$ and simplest solutions with unit matrix $\Lambda^{2}=I$, equation (1.5) has no other solutions. For the planar case, it was proven in [4]. In the planar case, the eigenvalues of matrix $\Lambda^{2}$ are equal to one: $D=I$. In the spatial case, the eigenvalues of matrix $\Lambda^{2}$ satisfy the relations $\lambda_{i}>0, \lambda_{1} \lambda_{2} \lambda_{3}=1$. By the separation theorem for singular numbers [5], the inequalities

$$
0<\lambda_{1} \leqslant 1 \leqslant \lambda_{2} \leqslant 1 \leqslant \lambda_{3}
$$

hold true. Hence, $\lambda_{2}=1, \lambda_{1}=d, \lambda_{3}=d^{-1}$ and $D=d e_{11}+e_{22}+d^{-1} e_{33}$. Hereinafter $e_{i j}$ is the $3 \times 3$ matrix whose entry at the intersection of $i^{\text {th }}$ row and $j^{\text {th }}$ column is one and all other entries are zero. 
As an independent variable, it is convenient to choose the values of function $d(t), d(0)=1$. Function $t(d)$ is inverse for function $d(t)$. Equations (3.5) become

$$
\begin{gathered}
2 \ddot{\Lambda}^{2} \dot{t}-2 \dot{\Lambda^{2}} \ddot{t}=\dot{t}\left(\dot{\Lambda}^{2}-2 \dot{t} E<\vec{\omega}>\right) \Lambda^{-2}\left(\dot{\Lambda}^{2}+2 \dot{t} E<\vec{\omega}>\right)+4 \dot{t}^{3} S_{0}, \\
\Lambda^{2}(1)=I, \quad \dot{\Lambda^{2}}(1)=2 t_{1} S_{1},
\end{gathered}
$$

where the dot stands for the derivative w.r.t. variable $d$.

Function $t(d)$ is represented by the series

$$
t=t_{1}(d-1)+\sum_{i \geq 2} t_{i}(d-1)^{i}
$$

As $t_{1} \neq 0$, change $t_{i} t_{1}^{-1} \rightarrow t_{i}, t_{1}^{2} \vec{\omega}_{0} \rightarrow \vec{\omega}_{0}, t_{1} \vec{\omega}_{1} \rightarrow \vec{\omega}_{1}, t_{1}^{2} S_{0} \rightarrow S_{0}, t_{1} S_{1} \rightarrow S_{1}$ makes $t_{1}=1$.

Matrix $\Lambda^{2}$ is represented as

$$
\begin{gathered}
\Lambda^{2}=O \operatorname{diag}\left(d, 1, d^{-1}\right) O^{T}=I+(d-1)\left(G_{1}-d^{-1} G_{3}\right), \\
\Lambda^{-2}=I+(d-1)\left(G_{3}-d^{-1} G_{1}\right),
\end{gathered}
$$

where $G_{k}=O e_{k k} O^{T}, G_{k}^{2}=G_{k}, O(1)=I, G_{1} G_{3}=0$,

$$
\dot{G}_{k}=\operatorname{ad}(\vec{\sigma}) G_{k}, \quad G_{k}(1)=e_{k k} .
$$

Condition (5.2) implies the identity $e_{11}-e_{33}=2 S_{1}$. As $d=1$, equation (5.1) determines the matrix

$$
S_{0}=E<\vec{\omega}_{1}>^{2}+\operatorname{ad}\left(\vec{\sigma}_{0}+2^{-1} \vec{\omega}_{1}\right)\left(e_{11}-e_{33}\right)-4^{-1} e_{11}+4^{-1} 3 e_{33}-t_{2}\left(e_{11}-e_{33}\right),
$$

where $\vec{\sigma}(1)=\vec{\sigma}_{0}$. Here we have used the standard notation for the commutator of matrices

$$
\operatorname{ad}(\vec{\sigma}) S=E<\vec{\sigma}>S-S E<\vec{\sigma}>.
$$

All the quantities involved in equation (5.1) are expressed via functions $t, \vec{\sigma}$ and parameters $\vec{\omega}_{0}, \vec{\omega}_{1}$ related by four scalar identities $S_{0} \vec{\omega}_{0}=0, \vec{\omega}_{0} \cdot \vec{\omega}_{1}=0$ :

$$
\begin{gathered}
\dot{\Lambda^{2}}=G_{1}-d^{-2} G_{3}+(d-1) \operatorname{ad}(\vec{\sigma})\left(G_{1}-d^{-1} G_{3}\right), \\
\ddot{\Lambda^{2}}=2 d^{-3} G_{3}+2 \operatorname{ad}(\vec{\sigma})\left(G_{1}-d^{-2} G_{3}\right)+(d-1)\left(\operatorname{ad}(\dot{\vec{\sigma}})+\operatorname{ad}(\vec{\sigma})^{2}\right)\left(G_{1}-d^{-1} G_{3}\right) .
\end{gathered}
$$

The solution to (5.1) is represented by the series

$$
t=d-1+\sum_{i \geq 2} t_{i}(d-1)^{i}, \quad \vec{\sigma}=\sum_{i \geq 0} \vec{\sigma}_{i}(d-1)^{i} .
$$

After substituting matrix (5.3), equation (5.1) can be written as

$$
\begin{aligned}
& 4 \dot{t}\left(d^{-3} G_{3}+\operatorname{ad}(\vec{\sigma})\left(G_{1}-d^{-2} G_{3}\right)\right)-2 \ddot{t}\left(G_{1}-d^{-2} G_{3}\right)-\dot{t}\left(G_{1}+d^{-4} G_{3}\right) \\
& +2 \dot{t}^{2} \operatorname{ad}\left(\vec{\omega}_{1}\right)\left(G_{1}-d^{-2} G_{3}\right)-4 \dot{t}^{3}\left[\operatorname{ad}\left(\vec{\sigma}_{0}+2^{-1} \vec{\omega}_{1}\right)\left(e_{11}-e_{33}\right)-4^{-1} e_{11}+4^{-1} 3 e_{33}-t_{2}\left(e_{11}-e_{33}\right)\right] \\
& =2 \ddot{t}(d-1) \operatorname{ad}(\vec{\sigma})\left(G_{1}-d^{-1} G_{3}\right)-2 \dot{t}(d-1)\left(\operatorname{ad}(\dot{\vec{\sigma}})+\operatorname{ad}(\vec{\sigma})^{2}\right)\left(G_{1}-d^{-1} G_{3}\right) \\
& +\dot{t}(d-1)\left[\operatorname{ad}(\vec{\sigma})\left(G_{1}+d^{-3} G_{3}\right)-d^{-2}(d-1)\left(G_{1} E<\vec{\sigma}>G_{3}-G_{3} E<\vec{\sigma}>G_{1}\right)\right] \\
& +\dot{t}(d-1)^{2}\left(\operatorname{ad}(\vec{\sigma})\left(G_{1}-d^{-1} G_{3}\right)\right)^{2}-2 \dot{t}^{2} t \operatorname{ad}\left(\vec{\omega}_{0}\right)\left[G_{1}-d^{-2} G_{3}+(d-1) \operatorname{ad}(\vec{\sigma})\left(G_{1}-d^{-1} G_{3}\right)\right] \\
& -2 \dot{t}(d-1) \operatorname{ad}\left(\vec{\omega}_{1}\right) \operatorname{ad}(\vec{\sigma})\left(G_{1}-d^{-1} G_{3}\right)-4 \dot{t}^{3} t\left(t E<\vec{\omega}_{0}>^{2}+\omega_{0} \otimes \omega_{1}+\omega_{1} \otimes \omega_{0}\right) \\
& +(d-1) \dot{t}\left[G_{1}-d^{-2} G_{3}+(d-1) \operatorname{ad}(\vec{\sigma})\left(G_{1}-d^{-1} G_{3}\right)-2 \dot{t} E<\vec{\omega}>\right]\left(G_{3}-d^{-1} G_{1}\right)\left[G_{1}-d^{-2} G_{3}\right. \\
& \left.+(d-1) \operatorname{ad}(\vec{\sigma})\left(G_{1}-d^{-1} G_{3}\right)+2 \dot{t} E<\vec{\omega}>\right] .
\end{aligned}
$$


Vanishing of the coefficient at $d-1$ in equation (5.8) gives the matrix identity

$$
\begin{aligned}
& -12\left(t_{3}-2 t_{2}^{2}\right)\left(e_{11}-e_{33}\right)-4 t_{2} \operatorname{ad}\left(5 \vec{\sigma}_{0}+\vec{\omega}_{1}\right)\left(e_{11}-e_{33}\right)+4 t_{2}\left(e_{11}-5 e_{33}\right) \\
& +\operatorname{ad}\left(\vec{\sigma}_{0}\right)\left(11 e_{33}-e_{11}\right)+4 \operatorname{ad}\left(\vec{\omega}_{1}\right) e_{33}+6\left(\operatorname{ad}\left(\vec{\sigma}_{1}\right)+\operatorname{ad}\left(\vec{\sigma}_{0}\right)^{2}\right)\left(e_{11}-e_{33}\right) \\
& -12 t_{3} \operatorname{ad}\left(\vec{\sigma}_{0}\right)\left(e_{11}-e_{33}\right)-\operatorname{ad}\left(\vec{\sigma}_{0}\right)\left(e_{11}+e_{33}\right)+2 \operatorname{ad}\left(\vec{\omega}_{0}\right)\left(e_{11}-e_{33}\right)+4 \operatorname{ad}\left(\vec{\omega}_{1}\right) \operatorname{ad}\left(\vec{\sigma}_{0}\right)\left(e_{11}-e_{33}\right) \\
& +4 \vec{\omega}_{0} \otimes \vec{\omega}_{1}+4 \vec{\omega}_{1} \otimes \vec{\omega}_{0}+e_{11}-9 e_{33}+2 \operatorname{ad}\left(\vec{\omega}_{1}\right)\left(e_{11}+e_{33}\right)+4 E<\vec{\omega}_{1}>\left(e_{33}-e_{11}\right) E<\vec{\omega}_{1}>=0 .
\end{aligned}
$$

The trace of this matrix identity determines the parameter

$$
t_{2}=-2^{-1}+4^{-1}\left(\left(\omega_{1}^{3}\right)^{2}-\left(\omega_{1}^{1}\right)^{2}\right) .
$$

The upper diagonal elements determine the parameter

$$
\begin{aligned}
12 t_{3}=5 & -\left(\omega_{1}^{3}\right)^{2}+\left(\omega_{1}^{1}\right)^{2}+2^{-1} 3\left(\left(\omega_{1}^{3}\right)^{2}-\left(\omega_{1}^{1}\right)^{2}\right)^{2}-12\left(\sigma_{0}^{1}\right)^{2}-24\left(\sigma_{0}^{2}\right)^{2} \\
& -8 \omega_{1}^{1} \sigma_{0}^{1}-16 \omega_{1}^{2} \sigma_{0}^{2}-8 \omega_{0}^{3} \omega_{1}^{3}-4\left(\omega_{1}^{2}\right)^{2} .
\end{aligned}
$$

Non-diagonal entries determine vector $\vec{\sigma}_{1}$. Middle diagonal entries give the equation

$$
3\left(\sigma_{0}^{3}\right)^{2}-3\left(\sigma_{0}^{1}\right)^{2}+2 \omega_{1}^{3} \sigma_{0}^{3}-2 \omega_{1}^{1} \sigma_{0}^{1}+2 \omega_{0}^{2} \omega_{1}^{2}+\left(\omega_{1}^{3}\right)^{2}-\left(\omega_{1}^{1}\right)^{2}=0 .
$$

The identity $S_{0} \vec{\omega}_{0}=0$ determines vector $\vec{\sigma}_{0}$ for $\omega_{0}^{1} \omega_{0}^{2} \omega_{0}^{3} \neq 0$ :

$$
\begin{aligned}
4 \omega_{0}^{1} \omega_{0}^{3}\left(\sigma_{0}^{2}+2^{-1} \omega_{1}^{2}\right)= & \vec{\omega}_{1}^{2}\left(\left(\omega_{0}^{2}\right)^{2}-\left(\omega_{0}^{1}\right)^{2}-\left(\omega_{0}^{3}\right)^{2}\right)+4^{-1}\left(\left(\omega_{0}^{1}\right)^{2}+\left(\omega_{0}^{3}\right)^{2}\right) \\
& +4^{-1}\left(\left(\omega_{0}^{1}\right)^{2}-\left(\omega_{0}^{3}\right)^{2}\right)\left(\left(\omega_{1}^{1}\right)^{2}-\left(\omega_{1}^{3}\right)^{2}\right), \\
\omega_{0}^{2}\left(\sigma_{0}^{1}+2^{-1} \omega_{1}^{1}\right)= & 2 \omega_{0}^{1}\left(\sigma_{0}^{2}+2^{-1} \omega_{1}^{2}\right)+\omega_{0}^{3}\left(\vec{\omega}_{1}^{2}-4^{-1}+4^{-1}\left(\omega_{1}^{1}\right)^{2}-4^{-1}\left(\omega_{1}^{3}\right)^{2}\right), \\
\omega_{0}^{2}\left(\sigma_{0}^{3}+2^{-1} \omega_{1}^{3}\right)= & 2 \omega_{0}^{3}\left(\sigma_{0}^{2}+2^{-1} \omega_{1}^{2}\right)+\omega_{0}^{1}\left(\vec{\omega}_{1}^{2}-4^{-1}-4^{-1}\left(\omega_{1}^{1}\right)^{2}+4^{-1}\left(\omega_{1}^{3}\right)^{2}\right) .
\end{aligned}
$$

Thus, we have two equations (5.9) and $\vec{\omega}_{0} \cdot \vec{\omega}_{1}=0$ for six parameters.

If $\omega_{0}^{2}=0, \omega_{0}^{1} \omega_{0}^{3} \neq 0$, by (5.10) we find $\sigma_{0}^{2}$ and we have three equations

$$
\begin{gathered}
\left(\left(\omega_{1}^{1}\right)^{2}-\left(\omega_{1}^{3}\right)^{2}\right)\left(4 \vec{\omega}_{1}^{2}-1+\left(\omega_{1}^{1}\right)^{2}+\left(\omega_{1}^{3}\right)^{2}\right)=0 \\
\omega_{0}^{1} \omega_{1}^{1}+\omega_{0}^{3} \omega_{1}^{3}=0 \\
3\left(\sigma_{0}^{3}\right)^{2}+2 \omega_{1}^{3} \sigma_{0}^{3}+\left(\omega_{1}^{3}\right)^{2}=3\left(\sigma_{0}^{1}\right)^{2}+2 \omega_{1}^{1} \sigma_{0}^{1}+\left(\omega_{1}^{1}\right)^{2}
\end{gathered}
$$

for seven parameters $\vec{\omega}_{1}, \omega_{0}^{1}, \omega_{0}^{3}, \sigma_{0}^{1}, \sigma_{0}^{3}$.

If $\omega_{0}^{2}=0, \omega_{0}^{1}=0, \omega_{0}^{2} \neq 0$, then $\sigma_{0}^{2}=-2^{-1} \omega_{1}^{2}, \omega_{1}^{3}=0$ and there remain two equations (5.11) and $5\left(\omega_{1}^{1}\right)^{2}+4\left(\omega_{1}^{2}\right)^{2}=1$ for five parameters $\sigma_{0}^{1}, \sigma_{0}^{3}, \omega_{0}^{3}, \omega_{1}^{1}, \omega_{1}^{2}$.

If $\vec{\omega}_{0}=0$, there is just one equation (5.11) for six parameters $\vec{\sigma}_{0}, \vec{\omega}_{1}$.

If $\omega_{0}^{2} \neq 0, \omega_{0}^{1}=0$ (or $\omega_{0}^{3}=0$ ), we can determine $\sigma_{0}^{1}, \sigma_{0}^{3}$ and there remain three equations $(5.9), \vec{\omega}_{0} \cdot \vec{\omega}_{1}=0$ and

$$
\left(\omega_{1}^{2}\right)^{2}\left(4 \vec{\omega}_{1}^{2}-1\right)=\left(4 \vec{\omega}_{1}^{2}+1\right)\left(\left(\omega_{1}^{3}\right)^{2}-\left(\omega_{1}^{1}\right)^{2}\right)
$$

for six parameters $\vec{\omega}_{1}, \omega_{0}^{2}, \omega_{0}^{3}$ (or $\left.\omega_{0}^{1}\right), \sigma_{0}^{2}$.

Finally, if $\omega_{0}^{1}=\omega_{0}^{3}=0, \omega_{0}^{2} \neq 0$, then $\vec{\omega}_{1}=0,\left(\sigma_{0}^{3}\right)^{2}=\left(\sigma_{0}^{2}\right)^{2}$. There is just one equation for four parameters $\vec{\sigma}_{0}, \omega_{0}^{2}$.

Vanishing of the coefficient at $(d-1)^{2}$ in equation (5.8) gives six scalar equations. At that, there appear new parameters $t_{4}, \vec{\sigma}_{2}$ which are identified uniquely. There remain two equations for the aforementioned parameters.

Vanishing of the coefficient at $(d-1)^{3}$ in equation (5.8) produces six new scalar equations. At that, there appear new parameters $t_{5}, \vec{\sigma}_{3}$, which are uniquely determined. There remain two equations for the aforementioned parameters.

Vanishing of the coefficient at $(d-1)^{4}$ in equation (5.8) implies six new scalar equations. At that, there appear new parameters $t_{6}, \vec{\sigma}_{4}$, which are uniquely determined. There remain two equations for the aforementioned parameters which overdetermine the algebraic system. 
At each step, vanishing of the coefficient at $(d-1)^{k}$ in equation (5.8) determines parameters $t_{(k+1)}, \vec{\sigma}_{k}$ and add two new equations for the aforementioned parameters.

Thus, we obtain an infinite chain of equations for the parameters whose total amount is six. This fact necessarily leads us to the contradiction.

The original proof of the contradiction is as follows. Equations $G_{k}^{2}=G_{k}, k=1,3$, have solutions $G_{k}=\vec{\lambda}_{k} \otimes \vec{\lambda}_{k}, \vec{\lambda}^{2}=1$. Condition $G_{1} G_{3}=0$ yields $\vec{\lambda}_{1} \cdot \vec{\lambda}_{3}=0$. Denoting $\vec{\lambda}_{2}=\vec{\lambda}_{3} \times \vec{\lambda}_{1}$, three vectors $\vec{\lambda}_{1}, \vec{\lambda}_{2}, \vec{\lambda}_{3}$ form a moving orthonormalized frame. Equations (5.5) provide the motion of this frame:

$$
\dot{\vec{\lambda}}_{k}=\vec{\sigma} \times \vec{\lambda}_{k}, \quad \vec{\lambda}_{k}(1)=\vec{e}_{k}, \quad k=1,2,3
$$

where

$$
\vec{e}_{1}=\left\|\begin{array}{l}
1 \\
0 \\
0
\end{array}\right\|, \quad \vec{e}_{2}=\left\|\begin{array}{l}
0 \\
1 \\
0
\end{array}\right\|, \quad \vec{e}_{3}=\left\|\begin{array}{l}
0 \\
0 \\
1
\end{array}\right\|,
$$

and $\vec{\sigma}$ is the angular velocity of the moving frame.

Equations (5.12) yield the identity

$$
2 \vec{\sigma}=\vec{\lambda}_{1} \times \dot{\vec{\lambda}}_{1}+\vec{\lambda}_{2} \times \dot{\vec{\lambda}}_{2}+\vec{\lambda}_{3} \times \dot{\vec{\lambda}}_{3}
$$

It follows from identities $G_{k}^{2}=G_{k}$ and (5.13) that functions $\vec{\lambda}_{k}, \vec{\sigma}$ has no poles for each value $d$.

The formulae

$$
\begin{aligned}
\operatorname{ad}(\vec{\sigma}) G_{k} & =\dot{\vec{\lambda}}_{k} \otimes \vec{\lambda}_{k}+\vec{\lambda}_{k} \otimes \dot{\vec{\lambda}}_{k} \\
\left(\operatorname{ad}(\dot{\vec{\sigma}})+\operatorname{ad}(\vec{\sigma})^{2}\right) G_{k} & =\ddot{\vec{\lambda}}_{k} \otimes \vec{\lambda}_{k}+\vec{\lambda}_{k} \otimes \ddot{\vec{\lambda}}_{k}+2 \dot{\vec{\lambda}}_{k} \times \dot{\vec{\lambda}}_{k},
\end{aligned}
$$

hold true, and due to them equation (5.8) becomes

$$
\begin{aligned}
& 2 d^{2} \ddot{t}\left[2 \vec{\lambda}_{3} \otimes \vec{\lambda}_{3}+2 d^{3}\left(\dot{\vec{\lambda}}_{1} \otimes \vec{\lambda}_{1}+\vec{\lambda}_{1} \otimes \dot{\vec{\lambda}}_{1}\right)-2 d\left(\dot{\vec{\lambda}}_{3} \otimes \vec{\lambda}_{3}+\vec{\lambda}_{3} \otimes \dot{\vec{\lambda}}_{3}\right)\right. \\
& \left.\quad+d^{3}(d-1)\left(\overrightarrow{\vec{\lambda}}_{1} \otimes \vec{\lambda}_{1}+\vec{\lambda}_{1} \otimes \ddot{\vec{\lambda}}_{1}\right)+d^{2}(d-1)\left(\ddot{\vec{\lambda}}_{3} \otimes \vec{\lambda}_{3}+\vec{\lambda}_{3} \otimes \ddot{\vec{\lambda}}_{3}+2 \dot{\vec{\lambda}}_{3} \times \dot{\vec{\lambda}}_{3}\right)\right] \\
& \quad-2 d^{3} \ddot{t}\left[d^{2} \vec{\lambda}_{1} \otimes \vec{\lambda}_{1}-\vec{\lambda}_{3} \otimes \vec{\lambda}_{3}+d^{2}(d-1)\left(\dot{\vec{\lambda}}_{1} \otimes \vec{\lambda}_{1}+\vec{\lambda}_{1} \otimes \dot{\vec{\lambda}}_{1}\right)-d(d-1)\left(\dot{\vec{\lambda}}_{3} \otimes \vec{\lambda}_{3}+\vec{\lambda}_{3} \otimes \dot{\vec{\lambda}}_{3}\right)\right] \\
& =\dot{t}\left[d^{2} \vec{\lambda}_{1} \otimes \vec{\lambda}_{1}-\vec{\lambda}_{3} \otimes \vec{\lambda}_{3}+d^{2}(d-1)\left(\dot{\vec{\lambda}}_{1} \otimes \vec{\lambda}_{1}+\vec{\lambda}_{1} \otimes \dot{\vec{\lambda}}_{1}\right)-d(d-1)\left(\dot{\vec{\lambda}}_{3} \otimes \vec{\lambda}_{3}+\vec{\lambda}_{3} \otimes \dot{\vec{\lambda}}_{3}\right)\right. \\
& \left.\quad-2 d^{2} \dot{t} E<t \vec{\omega}_{0}+\vec{\omega}_{1}>\right]\left(d I+(d-1)\left(d \vec{\lambda}_{3} \otimes \vec{\lambda}_{3}-\vec{\lambda}_{1} \otimes \vec{\lambda}_{1}\right)\left[d^{2} \vec{\lambda}_{1} \otimes \vec{\lambda}_{1}\right.\right. \\
& \quad-\vec{\lambda}_{3} \otimes \vec{\lambda}_{3}+d^{2}(d-1)\left(\dot{\vec{\lambda}}_{1} \otimes \vec{\lambda}_{1}+\vec{\lambda}_{1} \otimes \dot{\vec{\lambda}}_{1}\right)-d(d-1)\left(\dot{\vec{\lambda}}_{3} \otimes \vec{\lambda}_{3}+\vec{\lambda}_{3} \otimes \dot{\vec{\lambda}}_{3}\right) \\
& \left.\quad+2 d^{2} \dot{t} E<t \vec{\omega}_{0}+\vec{\omega}_{1}>\right]+4 d^{5} \dot{t}^{3} S_{0} .
\end{aligned}
$$

The coefficients at $t, \dot{t}, \ddot{t}$ have no poles at the point $d=0$. The leading terms of the asymptotics for these coefficients provide the approximate identity

$$
\left(\dot{t}+2^{-1} d \ddot{t}\right) \vec{\lambda}_{03} \otimes \vec{\lambda}_{03} \simeq-d^{2} \dot{t}^{3}\left[\left(\left(t \vec{\omega}_{0}+\vec{\omega}_{1}\right) \times \vec{\lambda}_{10}\right) \otimes\left(\left(t \vec{\omega}_{0}+\vec{\omega}_{1}\right) \times \vec{\lambda}_{10}\right)+d S_{0}\right],
$$

where $\vec{\lambda}_{k 0}=\vec{\lambda}_{k}(0)$.

Suppose that function $t(d)$ has a pole-like singularity at point $d=0: t=t_{-j} d^{-j}+\ldots, t_{-j} \neq 0$, $j>0$. Then identity (5.14) has the following leading terms:

$$
2^{-1} j(j-1) t_{-j} \vec{\lambda}_{30} \otimes \vec{\lambda}_{30} \simeq t_{-j}^{5} j^{3} d^{-4 j}\left(\vec{\omega}_{0} \times \vec{\lambda}_{10}\right) \otimes\left(\vec{\omega}_{0} \times \vec{\lambda}_{10}\right) .
$$

It implies the identity $\vec{\lambda}_{10}=k \vec{\omega}_{0}$. In view of the obtained relation, identity (5.14) has the following main terms:

$$
2^{-1} j(j-1) t_{-j} \vec{\lambda}_{30} \otimes \vec{\lambda}_{30} \simeq t_{-j}^{3} j^{3} d^{-2 j}\left(\vec{\omega}_{1} \times \vec{\lambda}_{10}\right) \otimes\left(\vec{\omega}_{1} \times \vec{\lambda}_{10}\right) .
$$


It implies the identity $\vec{\lambda}_{10}=l \vec{\omega}_{1}$. Scalar product of the obtained relation leads us to the contradiction:

$$
1=\vec{\lambda}_{10}^{2}=k l \vec{\omega}_{0} \cdot \vec{\omega}_{1}=0 .
$$

Thus, function $t(d)$ has no singularity at zero. Let $t=t_{0}+t_{k} d^{k}+\ldots, k>0$, then (5.14) has the following leading terms:

$$
2^{-1} k(k+1) t_{k} \vec{\lambda}_{03} \otimes \vec{\lambda}_{03} \simeq k^{3} t_{k}^{3} d^{2 k+2}\left[\left(\left(t_{0} \vec{\omega}_{0}+\vec{\omega}_{1}\right) \times \vec{\lambda}_{10}\right) \otimes\left(\left(t_{0} \vec{\omega}_{0}+\vec{\omega}_{1}\right) \times \vec{\lambda}_{10}\right)+d S_{0}\right] .
$$

It yields that $t_{k}=0, k>0$ and $t$ is constant, the contradiction.

\section{BIBLIOGRAPHY}

1. Yu.V. Tarasova. Classification of submodels with a linear velocity field in gas dynamics // Sibir. Zhurn. Industr. Matem. 12:4, 128-136 (2009). [J. Appl. Indust. Math. 12:4, 570-577 (2010).]

2. Yu.V. Yulmukhametova. Submodels of gas motion with a linear velocity field in the degenerate case // Sibir. Zhurn. Industr. Matem. 14:2, 139-150 (2011). [J. Appl. Indust. Math. 6:1, 123-133 (2012).]

3. L.V. Ovsyannikov. Lectures on foundation of gas dynamics. IKT, Moscow-Izhevsk (2003). (in Russian.)

4. S.V. Khabirov. Plane gas motions with the linear field of the velocity without divergence // Ufimskij Matem. Zhurn. 2:3, 107-113 (2010). (in Russian).

5. S.K. Godunov. Modern aspects of linear algebra. Nauchnaya kniga, Novosibirsk (1997). [Transl. Math. Mon. Amer. Math. Soc. Providence, RI 175. (1998).]

6. S.K. Godunov, E.I. Romenskii. Elements of continuus media mechanics and conservation laws. Novosibirsk, Nauchnaya kniga (1998).

Salavat Valeevich Khabirov, Institute of Mechanics USC RAS, October av., 71, 450054, Ufa, Russia

E-mail: habirov@anrb.ru 